\title{
Diferentes Intensidades Sobre a Capacidade Aeróbia e Produção de Lactato Pelo Músculo de Ratos Wistar
}

CIÊNCIAS DO EXERCÍCIO E DO ESPORTE

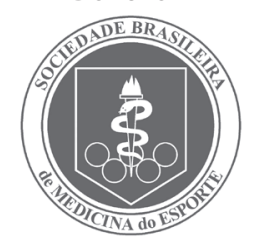

Artigo Original

\section{Running Training Effects in Different Intensities on the Aerobic Capacity and Lactate Production by the Muscle of Wistar Rats}

Michel Barbosa de Araújo

Fúlvia de Barros Manchado-

Gobatto $^{2}$

Fabrício Azevedo Voltarelli1,3

Carla Ribeiro'

Clécia Soares de Alencar Mota'

Claudio Alexandre Gobatto

Maria Alice Rostom de Mello'

1. Departamento de Educação Física, IB, Unesp, Rio Claro, SP, Brasil.

2. Faculdades Integradas Einstein de Limeira.

3. União das Faculdades de Dracena - Unifada.

Endereço para correspondência: Michel Barbosa de Araújo

Avenida 2 A, 868, Bela Vista

- 13506-780 - Rio Claro, SP, Brasil.

E-mail: mbujo@ig.com.br

Submetido em: 12/08/2009 Versão final recebida em: 04/05/2009 Aceito em: 05/05/2009

\section{RESUMO}

São raros os estudos que associam indicadores de capacidade aeróbia e os substratos produzidos pelo metabolismo muscular em ratos. Dessa forma, o objetivo do presente estudo foi verificar o efeito do treinamento de corrida em duas diferentes intensidades sobre a capacidade aeróbia e a produção de lactato pelo músculo sóleo isolado de ratos. Ratos Wistar (90 dias) tiveram a transição metabólica aeróbio-anaeróbia determinada pelo teste de máxima fase estável de lactato (MFEL). Em seguida, os ratos foram treinados 40 minutos/dia, cinco dias/semana, na velocidade equivalente à MFEL (TT) ou 5\% superior a essa (TS), por oito semanas. Como controles foram usados ratos mantidos sedentários (S). Ao final, todos os animais foram sacrificados para análise da produção de lactato pelo músculo sóleo isolado. No inicio do experimento, a maior parte dos animais obteve a MFEL na velocidade de $25 \mathrm{~m} / \mathrm{min}$ à concentração de 4,38 \pm _0,22 $\mathrm{mmol} / \mathrm{L}$ sanguínea de lactato. Ao final do experimento, a maior parte dos ratos treinados na TT apresentou MFEL na velocidade de $25 \mathrm{~m} / \mathrm{min}$, à concentração sanguínea de lactato $3,10 \pm 0,27 \mathrm{mmol} / \mathrm{L}$. A maioria dos treinados TS teve MFEL na velocidade de $25 \mathrm{~m} / \mathrm{min}$ à concentração sanguínea de lactado de $3,36 \pm 0,62 \mathrm{mmol} / \mathrm{L}$. Os sedentários mostraram a MFEL na velocidade de $20 \mathrm{~m} / \mathrm{min}$ à concentração sanguínea de lactato de 4,83 $\pm 0,67 \mathrm{mmol} / \mathrm{L}$. A produção de lactato ( $\mu \mathrm{mol} / \mathrm{g}$.h) pelo músculo sóleo isolado foi menor no grupo TS (3,83 $\pm 0,62)$ do que nos demais $(S 4,31 \pm 0,58$ e TT 4,71 $\pm 0,39$ ). A partir dos resultados obtidos no presente estudo, pode-se concluir que o treinamento aeróbio evitou a deterioração do condicionamento aeróbio imposta pelo avanço da idade e que o treinamento físico na intensidade superior à MFEL reduziu a produção muscular de lactato.

Palavras-chave: limiar anaeróbio, glicogênio, exercício físico.

\section{ABSTRACT}

There are few studies that associate indicators of aerobic capacity and the substrates produced by the muscular metabolism in rats. The aim of the present study was to analyze the effects of physical training in different intensities on the aerobic capacity and lactate production by the isolated soleus muscle of Wistar rats (90 days) that had the aerobic/anaerobic metabolic transition determined by the Maximal Lactate Steady State Test (MLSS). Subsequently, the rats were trained 40 minutes/day, 5 days/week, in the speed equivalent to MLSS (MT) or 5\% above it (AT), for 8 weeks. Rats maintained sedentary (S) were used as controls. At the end, all rats were sacrificed for analysis of lactate production by the isolated soleus muscle. The main results were: in the beginning of the experiment, in most of the rats the MLSS was obtained in the speed of $25 \mathrm{~m} / \mathrm{min}$, to the concentration of $4.38 \pm 0.22 \mathrm{mmol} / \mathrm{L}$ of blood lactate. At the end of the experiment, most of the rats trained at the MLSS intensity presented MLSS in the speed of $25 \mathrm{~m} / \mathrm{min}$, to the concentration of $3.10 \pm 0.27$ $\mathrm{mmol} / \mathrm{L}$ of blood lactate. Most of the animals trained above-MLSS had MLSS in the speed of $25 \mathrm{~m} / \mathrm{min}$, to the concentration of $3.36 \pm 0.62 \mathrm{mmol} / \mathrm{L}$ of blood lactate. Sedentary rats showed MLSS in the speed of $20 \mathrm{~m} / \mathrm{min}$ to the concentration of blood lactate of $4.83 \pm 0.67 \mathrm{mmol} / \mathrm{L}$. The lactate production $(\mu \mathrm{mol} / \mathrm{g} . \mathrm{h}): S 4.31 \pm 0.58$, MT $4.71 \pm 0.39$, AT $3.83 \pm 0.62$ was lower in the ST group., It can be concluded from the results of the present study that the aerobic training prevented the deterioration of the aerobic conditioning imposed by the age advance, and that physical training above the MLSS reduced muscle lactate production.

Keywords: anaerobic threshold, glycogen, physical exercise. 


\section{INTRODUÇÃO}

O fornecimento de adenosina trifosfato (ATP) para a manutenção do exercício pode ser proveniente dos metabolismos aeróbio e anaeróbio. Durante o exercício moderado, as respostas fisiológicas estabilizam-se rapidamente e o oxigênio supre de maneira satisfatória a demanda energética. Em intensidades mais elevadas, a via metabólica predominante é a anaeróbia, o que resulta na produção excessiva de lactato. Isso dificulta a manutenção do exercício por tempo prolongado, já que ocorrem a inibição da atividade enzimática e redução da atividade do cálcio e troponina ${ }^{(1)}$.

Existe uma zona de transição a partir da qual ocorre a mudança da predominância aeróbia para a anaeróbia, sendo essa zona de exercício extremamente importante para o condicionamento físico, treinamento e rendimento desportivo. Por essa razão, diversas investigações acerca dessa zona de transição vêm sendo realizadas nas últimas décadas, resultando em diferentes protocolos de avaliação(2-5). Dentre os mais utilizados, destacam-se o limiar ventilatório observado(2), a concentração de lactato sanguíneo para identificar o limiar anaeróbio (LAN), proposto por Kinderman et al.(3), o limiar anaeróbio obtido pela concentração fixa de 4,0mm (Onset of Blood Lactate Acumulation - OBLA), inicialmente sugerido por Sjodin e Jacobs ${ }^{(4)}$, o modelo de potência crítica não evasiva e exaustiva proposto por Monod e Scherer ${ }^{(5)}$, entre outros.

Grande parte desses protocolos de avaliação utiliza a resposta do lactato sanguíneo, devido à fidedignidade dessa variável sanguínea na mensuração da intensidade de transição metabólica bem como excelente resposta ao treinamento físico, permitindo, além da caracterização do esforço, acompanhamento da eficiência do treinamento.

O limiar anaeróbio (LAN) foi definido como a carga de trabalho na qual o lactato sanguíneo começa a se acumular desproporcionalmente durante o exercício, com cargas progressivas ${ }^{(2)}$ e, teoricamente, indica a máxima fase estável de lactato $(\mathrm{MFEL})^{(6)}$. A MFEL equivale à mais alta concentração sanguínea de lactato onde sua entrada na circulação é compensada pela remoção durante o exercício com carga constante $^{(7)}$.

A MFEL já foi utilizada para determinação da transição metabólica em ratos. Gobatto et al.(8) desenvolveram um estudo para a determinação do MFEL de ratos durante o exercício de natação. Nesse estudo, a MFEL foi obtida na carga de $6 \%$ do peso corporal à concentração sanguínea de lactato foi de $5,5 \mathrm{mmol} / \mathrm{I}$ de sangue. Mais recentemente, nosso grupo descreveu, também, um protocolo para determinação da MFEL de ratos durante exercício de corrida em esteira rolante. Nesse caso, a MFEL foi obtida na velocidade de $20 \mathrm{~m} / \mathrm{min}$, à concentração de lactato sanguíneo 3,9mmol/L ${ }^{(9)}$. Contudo, apenas o teste de MFEL não deixa claro o quanto de lactato foi produzido e transportado do músculo para a circulação sanguínea durante o treinamento.

Estudos envolvendo seres humanos sugerem que indivíduos treinados apresentaram aumento na capacidade de transporte do lactato da fibra muscular para a circulação sanguínea. Jacobs ${ }^{(10)}$ relatou que indivíduos treinados apresentaram aumento na capacidade de transporte do lactato da fibra muscular para a circulação sanguínea. Essas observações também foram descritas por Oyono-Enguelle et al. ${ }^{(11)} \mathrm{em}$ seres humanos e por Gobatto ${ }^{(12)}$ em ratos, sendo que os mesmos associaram o aumento da concentração de lactato sanguíneo, e menor concentração de lactato no músculo, ao maior efluxo muscular desse substrato durante o exercício físico agudo envolvendo indivíduos bem condicionados.

Desse modo, o objetivo do presente estudo foi verificar o efeito do treinamento de corrida em duas diferentes intensidades sobre a capacidade aeróbia, estimada pelo teste de MFEL e a produção de lactato pelo músculo sóleo isolado em ratos.

\section{MÉTODOS}

\section{Animais}

Foram utilizados ratos da linhagem Wistar com 90 dias que pesavam entre 320 e $400 \mathrm{~g}$ no inicio e 420 a 500g no final do experimento, que tiveram livre acesso à água e o alimento (ração comercial para roedores). Os animais foram mantidos em gaiolas coletivas de polietileno, medindo $37,0 \times 31,0 \times 16,0 \mathrm{~cm}$, (cinco animais por gaiola) sob condições de temperatura $\left(25^{\circ} \mathrm{C}\right)$ e ciclo claro/escuro (12h/12h), com as luzes acesas da $18 \mathrm{~h}$ às $6 \mathrm{~h}$. Todos os experimentos envolvendo animais foram realizados de acordo com as resoluções brasileiras específicas sob a bioética em experimento com animais (Lei no. 6.638, de 8 de maio de 1979 e Decreto no. 26.645 de 10 e julho de 1934).

\section{Protocolo experimental}

Inicialmente, os animais foram separados, selecionados e adaptados à esteira rolante no qual posteriormente foram avaliados.

\section{Seleção dos ratos corredores e adaptação à esteira rolante}

Previamente ao experimento, foi feita uma seleção dos ratos naturalmente "corredores". Nas três semanas que antecederam o período de treinamento, os animais foram adaptados à corrida em esteira, em velocidades e tempos progressivos conforme protocolo descrito na tabela 1, para posteriormente terem a transição metabólica aeróbioanaeróbia avaliada através da determinação da MFEL.

Tabela 1. Protocolo de adaptação à corrida em esteira rolante.

\begin{tabular}{c|c|c}
\hline Semanas & Velocidade $(\mathbf{m} / \mathbf{m i n})$ & Tempo $(\mathbf{m i n})$ \\
\hline 1 & 10 & 5 a 10 \\
\hline 2 & 15 a 20 & 5 a 10 \\
\hline 3 & 25 & 10 a 20 \\
\hline
\end{tabular}

\section{Determinação da MFEL}

Para determinação da MFEL foram realizadas séries de exercícios de 25 minutos de corrida em esteira rolante, a diferentes velocidades fixas a cada série, com intervalos de 48 horas entre elas e coleta de sangue $(25 \mu \mathrm{L})$ a cada cinco minutos, para dosagem de lactato. As coletas de sangue foram realizadas a partir de pequeno corte na extremidade da cauda do animal. Uma só incisão, efetuada antes do início do exercício, foi suficiente para a coleta de todas as amostras. A concentração sanguínea de lactato representativa da MFEL foi considerada na maior velocidade onde não ocorreu variação do lactato sanguíneo superior a 1,0mmol/L entre 10 e 25 min de exercício. A concentração de lactato sanguínea foi determinada por método enzimático ${ }^{(13)}$.

\section{Delineamento dos grupos e protocolos de exercício}

Os animais foram distribuídos em três grupos:

- Treinados na intensidade da máxima fase estável de lactato (TT): ratos $(n=10)$ submetidos ao treinamento de corrida em esteira rolante, na velocidade equivalente à da transição metabólica aeróbio-anaeróbia, 40 min por dia, cinco dias por semana, durante oito semanas;

- Treinados acima da máxima fase estável de lactato (TS): ratos ( $n=$ 10) submetidos ao treinamento em velocidade $5 \%$ superior à transição metabólica aeróbio-anaeróbia, 40 min por dia, cinco dias por semana, durante oito semanas.

- Sedentários (S): composto por ratos $(n=10)$ que não foram submetidos a treinamento.

\section{Avaliação do condicionamento aeróbio}

Na metade e no final do período experimental, os animais foram submetidos a uma sessão de corrida em esteira na velocidade equi- 
valente à MFEL por 25 minutos. Foram coletadas amostras de sangue, através de corte na extremidade distal da cauda, a cada cinco minutos de exercício para determinação das concentrações de lactato por analisador eletroquímico (modelo YSI 1500 Sport, Yellow Springs, OH, EUA).

\section{Produção de lactato pelo músculo sóleo isolado}

Quarenta e oito horas após a realização da última sessão de exercício (no caso dos grupos treinados), os animais foram anestesiados com tiopental sódico (40mg/kg de peso corporal, intraperitoneal) e, logo após, o músculo sóleo direito foi isolado com o mínimo de lesão possível e fatias longitudinais pesando entre 25 e $35 \mathrm{mg}$ foram inicialmente incubadas por 30 minutos a $37^{\circ} \mathrm{C}$ em banho-maria Dubinoff, dentro de frascos de vidro de cintilação contendo 1,5ml de tampão Krebs-Kinger-Bicarbonato ( $\mathrm{NaCl} 0,6 \%$, HEPES 6,64 mM; $\mathrm{Kcl} 0,032 \% ; \mathrm{CaCl}_{2} 1,14 \mathrm{mM} ; \mathrm{KH}_{2} \mathrm{PO}_{4} 0,015 \%$; $\mathrm{NaHCO}_{3} 0,19 \% ; \mathrm{MgSO}_{4} 0,03 \%$ ) e piruvato $5 \mathrm{mM}$, equilibrado com mistura gasosa $95 \mathrm{O}_{2}-5 \% \mathrm{CO}_{2}$, pH 7,4. Após esse período, as fatias foram transferidas para novo frasco de cintilação de vidro, contendo $1,5 \mathrm{ml}$ de tampão Krebs-Kinger-Bicarbonato, enriquecido com 5,5mM de glicose, contendo $\left(U^{14} \mathrm{C}\right)$ glicose $0,25 \mathrm{mCi} / \mathrm{mL}$ e $100 \mu \mathrm{U}$ de insulina. Em seguida o frasco foi fechado por uma membrana de borracha e por uma tampa plástica.

O sistema contendo as fatias musculares foi incubado em banhomaria Dubinoff por 60 minutos e o lactato radioativo liberado no meio de incubação foi determinado por separação de metabólitos em coluna de troca iônica (Dowex-2, Sigma), sendo a radioatividade medida em contador de partículas beta (modelo Tricarb 2100TR, Packard, São Paulo - SP, Brasil).

\section{ANÁLISE ESTATÍSTICA}

A análise estatística foi feita por ANOVA one way e aplicação do teste post-hoc de Newman-Keuls, onde adequado, com nível de significância prefixado em $5 \%$.

\section{RESULTADOS}

Os valores de lactato sanguíneo durante teste de esforço para determinação da máxima fase estável de lactato (MFEL) encontrados no início do experimento, referentes a um rato, a título de exemplo (figura 1), para esse animal, a MFEL ocorreu na velocidade de $25 \mathrm{~m} / \mathrm{min}$ à concentração de 4,11 $\pm 0,53 \mathrm{mmol} / \mathrm{L}$ sanguínea de lactado. Considerando todo o lote de animais avaliados, $82 \%$ dos animais obtiveram a

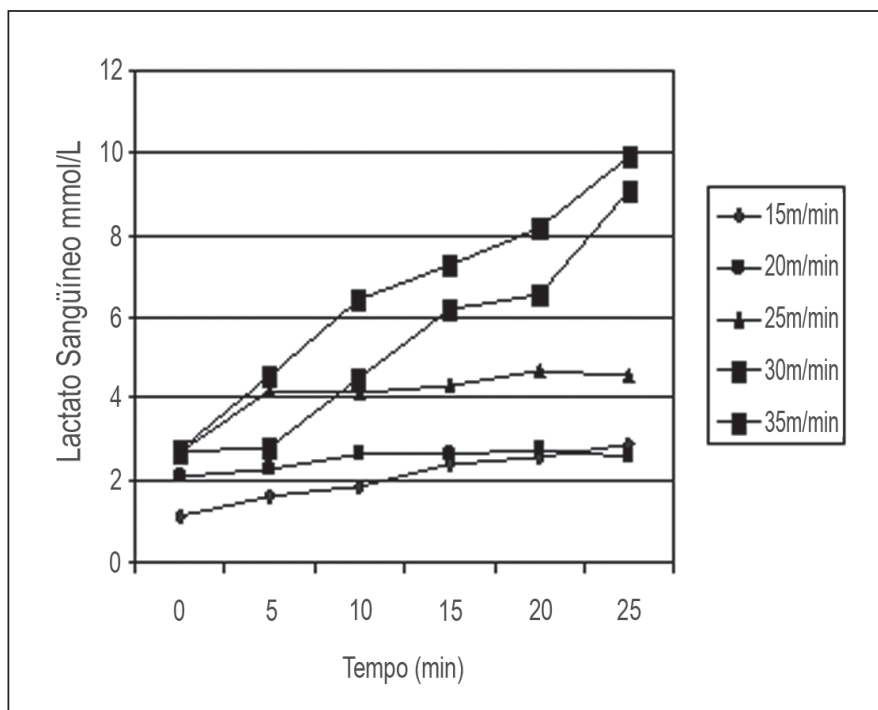

Figura 1. Lactato sanguíneo de um animal, a titulo de exemplo, durante teste de esforço para determinação da máxima fase estável de lactato no inicio do experimento. Para esse animal, a MFEL ocorreu na velocidade de $25 \mathrm{~m} / \mathrm{min}$ à concentração sanguínea de lactato de $4,11 \pm 0,53 \mathrm{mmol} / \mathrm{L}$.
MFEL na velocidade de $25 \mathrm{~m} / \mathrm{min}$ à concentração de $4,38 \pm 0,22 \mathrm{mmol} / \mathrm{L}$ sanguínea de lactato e 17\% tiveram tal parâmetro na velocidade de $30 \mathrm{~m} / \mathrm{min}$ à concentração de $4,42 \pm 0,45 \mathrm{mmol} / \mathrm{L}$ sanguínea de lactato. Os valores de lactato sanguíneo durante o teste de esforço para determinar a MFEL no final do experimento, referentes a um rato de cada grupo, a título de exemplo (figura 2), sendo que, considerando todo o lote de animais sedentários avaliados ao final do experimento, a maior

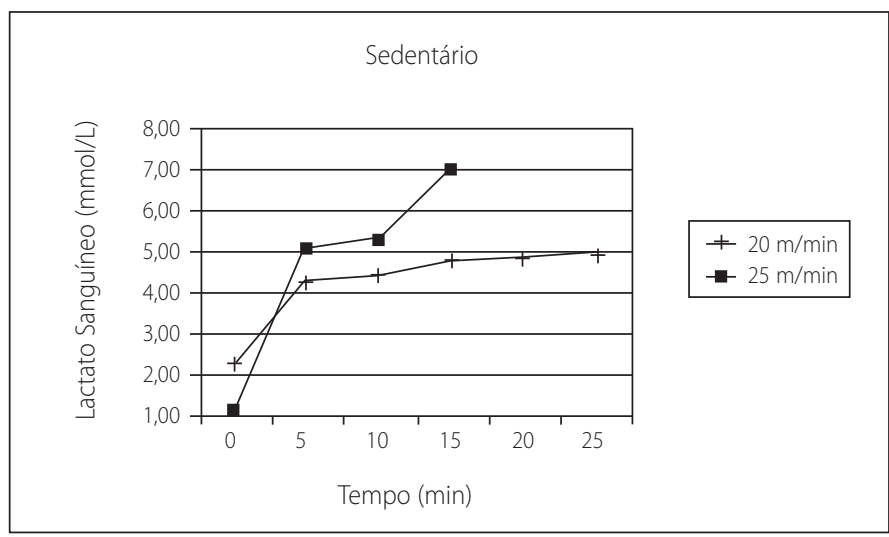

Figura 2A

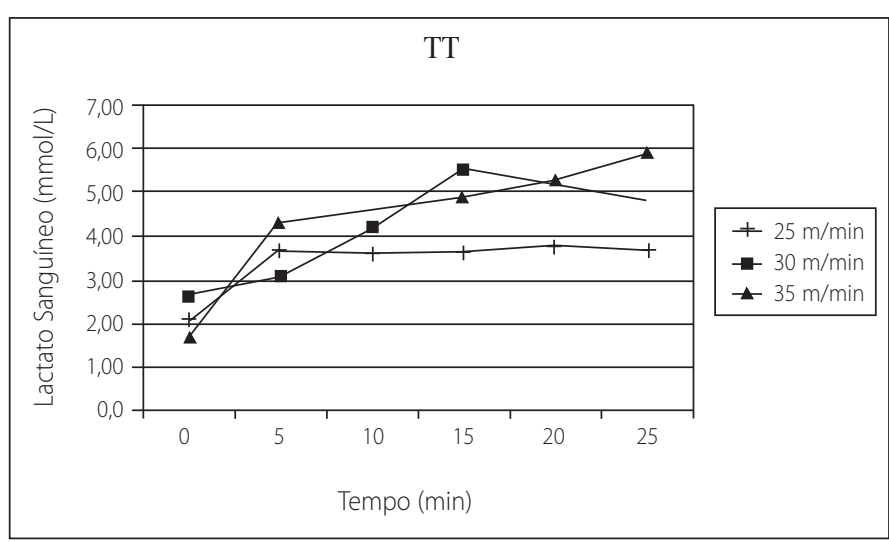

Figura 2B

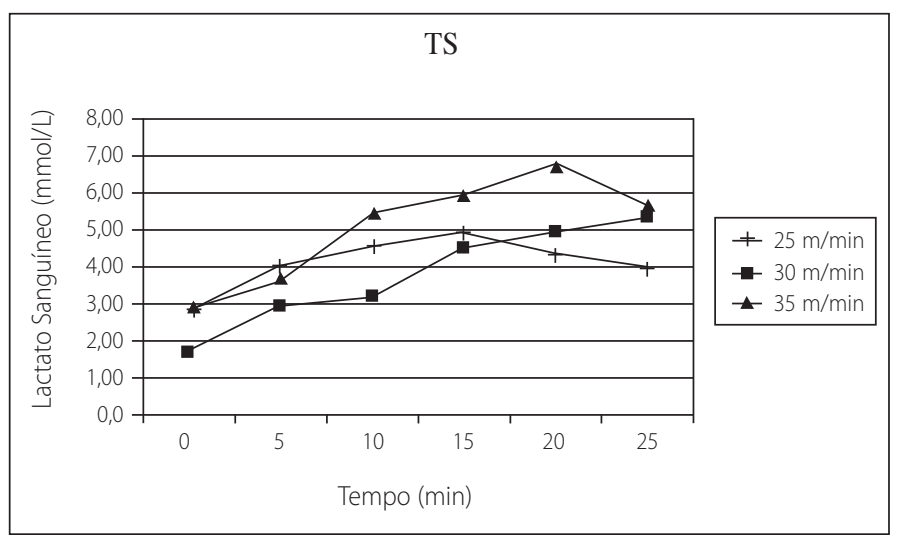

Figura $2 \mathrm{C}$

Figura 2. Lactato sanguíneo de um animal, a titulo de exemplo, durante teste de esforço para determinação da máxima fase estável de lactato no final do experimento. A) Grupo S; B) Grupo TT; e C) Grupo TS. Para o rato do grupo S a MFEL ocorreu na velocidade de $20 \mathrm{~m} / \mathrm{min}$ à concentração sanguínea de lactato de $4,90 \pm 1,02 \mathrm{mmol} / \mathrm{L} ; \mathrm{O}$ animal não suportou a realização do exercício na velocidade de $35 \mathrm{~m} / \mathrm{min}$. Para o rato do grupo TT, ocorreu na velocidade de $25 \mathrm{~m} / \mathrm{min}$ à concentração de $3,37 \pm 0,67 \mathrm{mmol} / \mathrm{L}$ Para o animal do grupo TS, ocorreu na velocidade de $25 \mathrm{~m} / \mathrm{min}$ à concentração de $4,16 \pm 0,74 \mathrm{mmol} / \mathrm{L}$ 
parte dos animais (72,72\%) obteve a MFEL na velocidade de $20 \mathrm{~m} / \mathrm{min}$ à concentração de 4,83 \pm 0,67mmol/L sanguínea de lactato. Em relação aos treinados na intensidade de transição metabólica (TT), a maior parte dos animais $(63,63 \%)$ obteve a MFEL na velocidade de $25 \mathrm{~m} / \mathrm{min}$ à concentração de 3,10 \pm 0,27 mmol/L sanguínea de lactato. Quanto aos treinados na intensidade superior à TS, a maior parte dos animais (54,54\%) obteve a MFEL na velocidade de $25 \mathrm{~m} / \mathrm{min}$ à concentração de 3,36 $\pm 0,62 \mathrm{mmol} / \mathrm{L}$ sanguínea de lactato.

Os valores referentes à diferença de concentração de lactato sanguíneo (tabela 2) entre o início e final do experimento, os animais mantidos sedentários apresentaram a média de concentração de 4,86 $\pm 1,30 \mathrm{mmol} / \mathrm{L}$ sanguíneos lactato no inicio do experimento. No final do experimento, os animais desse grupo apresentaram a concentração de 4,83 \pm 0,67 mmol/L mostrando, assim, diminuição de 0,03 mmol/L na concentração sanguínea de lactato. Em relação aos animais do grupo TT, estes apresentaram a concentração de 3,93 \pm 0,49 mmol/L sanguínea de lactado no inicio do experimento e concentração de 3,10 $\pm 0,27 \mathrm{mmol} / \mathrm{L}$ ao final do experimento, havendo, assim, diminuição de $0,83 \mathrm{mmol} /$ na concentração L sanguínea de lactato. Quanto aos animais do grupo TS, os mesmos apresentaram a concentração de $3,61 \pm 0,62 \mathrm{mmol} / \mathrm{L}$ no inicio do experimento e de 3,36 \pm 0,62 $\mathrm{mmol} / \mathrm{L}$ ao final do experimento, havendo assim, diminuição de 0,25 mmol/L na concentração sanguínea de lactado.

A produção de lactado pelo músculo sóleo apresentou diminuição significativa no grupo de animais TS em relação aos animais TT, em ambas as intensidades (figura 3).

Tabela 2. Concentração sanguínea de lactato ( $\mathrm{mmol} / \mathrm{L}$ ) em repouso no inicio e no final do experimento.

\begin{tabular}{l|c|c|c}
\hline & Sedentário (10) & TT (6) & TS (7) \\
\hline $\begin{array}{l}\text { Concentração lactato sanguíneo } \\
\text { no início do experimento }\end{array}$ & $4,86 \pm 1,30$ & $3,93 \pm 0,49^{\mathrm{b}}$ & $3,61 \pm 0,52^{\mathrm{a}}$ \\
\hline $\begin{array}{l}\text { Concentração de lactato sanguí- } \\
\text { neo no final do experimento }\end{array}$ & $4,83 \pm 0,67$ & $3,10+0,27^{\mathrm{b}}$ & $3,36+0,62^{\mathrm{a}}$ \\
\hline \begin{tabular}{l} 
Diferença entre início e o final \\
\hline
\end{tabular} & $0,03 \pm 0,02$ & $0,83+0,59^{\mathrm{b}}$ & $0,25+0,18^{\mathrm{a}, \mathrm{c}}$ \\
\hline
\end{tabular}

Resultados expressos com média + desvio padrão, com o número de animais entre parênteses. TT = Animais treinados na intensidade da Máxima Fase Estável de Lactato. TT = Animais treinados em intensidade 5\% superior à Máxima Fase Estável de Lactato. Diferenças significativas (ANOVA $p<0,05 /$ teste de Newman-Keuls) a-Sedentário $\times$ TS, b-Sedentário $\times$ TT, c - TS $\times$ TT

\section{Produção de Lactato}

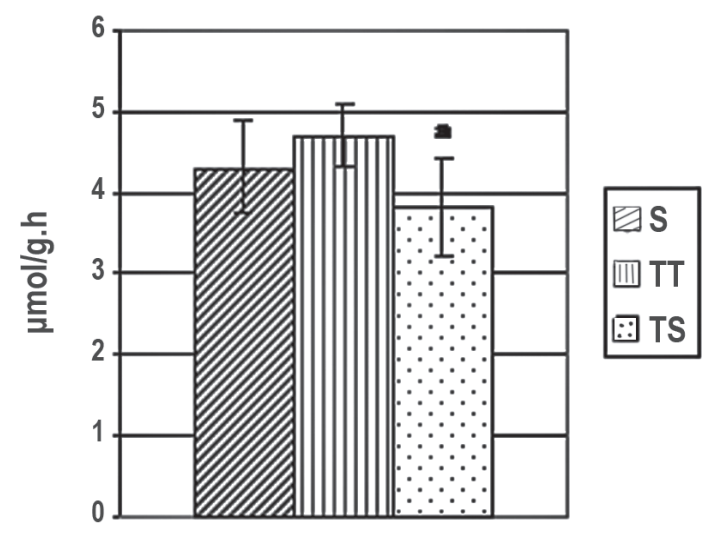

Figura 3. Produção de lactato no músculo sóleo dos animais ao final do experimento. Diferenças significativas (ANOVA $p<0,05 /$ teste de Newman-Keuls) a - TS x TT.

\section{DISCUSSÃO}

A concentração sanguínea de lactato foi bem definida nos últimos anos como um excelente indicador da predominância de fornecimento energético para o exercício realizado ${ }^{(13)}$. Desde então, tem sido muito utilizada para a avaliação, determinação da intensidade e prescrição de treinamento físico para humanos e animais.

Nos últimos anos, o protocolo de MFEL tem sido muito utilizado na determinação dessas concentrações sanguíneas de lactato, uma vez que esse método é considerado como "padrão ouro" para determinar a intensidade correspondente à zona de transição aeróbio-anaeróbia ${ }^{(14-16)}$. Porém, as concentrações de lactato verificadas nos estudo envolvendo corrida em esteira rolante não se apresentam unânimes, sendo o treinamento um fator determinante na alteração da produção e remoção de lactato no músculo.

No presente estudo, as análises dos resultados referentes aos testes de MFEL, efetuados no inicio do experimento, evidenciaram que, em ratos Wistar submetidos ao exercício físico em esteira rolante, o lactato sanguíneo apresenta um padrão semelhante àquele descrito para seres humanos. Resultados similares foram observados, recentemente, ao se utilizarem ratos submetidos ao exercício de natação e de corrida em esteira rolante ${ }^{(8,9,17)}$. A concentração média de lactato sanguíneo equivalente à MFEL, para todos os animais avaliados no início de nosso experimento, foi de 4,38 \pm 0,22 mmol/L e está de acordo com estudos prévios, os quais aplicaram o teste da MFEL em corrida em esteira rolante em animais sedentários e eutróficos ${ }^{(9,17)}$.

Para avaliar a eficácia do protocolo de treinamento como uma ferramenta útil na melhoria do condicionamento aeróbio dos animais, foi analisada, ao final do experimento, a concentração de lactato sanguíneo dos animais pertencentes aos grupos sedentários, TT e TS durante a realização de um segundo teste visando à determinação da MFEL. Os valores de lactado sanguíneo, observados nesse teste, mostraram-se mais elevados no grupo Sedentário quando comparado com os grupos treinados TT e TS, não sendo observadas diferenças entre os dois últimos. No que diz respeito aos valores de velocidade de corrida equivalentes à MFEL, estes foram menores nos sedentários em relação aos treinados TT e TS, sem diferenças entre este dois últimos.

Durante o exercício, a concentração sanguínea de lactato é dependente da razão entre a velocidade em que esse substrato é produzido pelo músculo esquelético e a velocidade com que o mesmo é removido da corrente sanguínea(18). Os mecanismos envolvidos no acúmulo de lactato durante o exercício são diversos, sendo o aumento da intensidade do exercício uma de suas principais causas ${ }^{(1)}$.

No exercício de baixa ou moderada intensidade, em seres humanos e ratos, o lactato sanguíneo permanece estável(1). Nessa situação, a taxa de produção de lactato apresenta equilíbrio ou mesmo mostrase inferior à de sua remoção ${ }^{(19)}$. Quando indivíduos são submetidos a exercícios de alta intensidade, a concentração sanguínea de lactato eleva-se após três a quatro minutos de atividade, indicando, assim, que a taxa de produção supera a velocidade de remoção(18). Diferentemente do exercício agudo, o treinamento, principalmente de natureza aeróbia, gera adaptações metabólicas consideráveis em relação ao turnover de lactato e, como consequência, ocasiona a redução de acúmulo do mesmo na corrente sanguínea durante o exercício para uma mesma intensidade submáxima de esforço ${ }^{(8,20)}$

Uma das causas da diminuição da concentração de lactato sanguíneo após o treinamento aeróbio é a redução na produção, consequente à maior oxidação de ácidos graxos e redução da glicólise anaeróbia(21) 
Por outro lado, alguns estudos têm demonstrado que a redução da concentração de lactato sanguíneo em exercício submáximo é determinada pela maior remoção de lactato. $\mathrm{O}$ aumento da remoção de lactato, verificada tanto em ratos ${ }^{(20)} \operatorname{como} \mathrm{em}$ seres humanos ${ }^{(22)}$, é realizado pelo fígado, rins coração e fibras musculares tipo I(23). A remoção da maior parte do lactato deve-se à oxidação e o restante, à conversão ao glicogênio (24).

Corroborando o achado na literatura, no presente estudo encontrou-se redução da liberação de lactato pelo músculo sóleo isolado em decorrência do treinamento em intensidade superior à da MFEL pelo músculo esquelético. Estudos envolvendo seres humanos sugerem que indivíduos treinados apresentaram aumento na capacidade de transporte do lactato da fibra muscular para a circulação sanguínea. Jacobs ${ }^{(25)}$, em estudos envolvendo seres humanos, relatou que indivíduos treinados apresentaram aumento na capacidade de transporte do lactato da fibra muscular para a circulação sanguínea.

Roth ${ }^{(26)}$, em estudos com ratos submetidos a diferentes treinamentos de velocidade e resistência muscular, verificou aumento da atividade de transporte do lactato para fora do tecido muscular. Sendo assim, era de se esperar que houvesse aumento dos valores do lactato liberado para o meio durante a incubação do músculo sóleo, fato que não foi observado no presente estudo.

Os resultados de produção de lactato pelo músculo sóleo isolado mostraram valores menores pelo grupo TS quando comparado com os grupos TT e Sedentário. Uma vez que os animais treinados na intensidade equivalente à transição metabólica (TT) apresentaram respostas semelhantes às dos animais do grupo sedentário nesse mesmo parâmetro, podemos sugerir que o treinamento físico na intensidade superior à máxima fase estável de lactato exerceu influência sobre o metabolismo glicolítico dos animais. Isso pode indicar diminuição da metabolização da glicose pela via anaeróbia lática no grupo TS ou, ainda, um possível redirecionamento desse substrato para a ressíntese de glicose e, consequentemente, de glicogênio muscular.

Além disso, os resultados dos testes de esforço efetuados após o treinamento aeróbio mostraram que o protocolo utilizado no presente estudo levou a redução do acúmulo de lactato sanguíneo durante o exercício. Isso indica que o protocolo de corrida por nós utilizado mostrou-se eficaz em melhorar o condicionamento aeróbio dos animais ou, ao menos, evitar sua redução consequente devido ao avanço etário dos animais.

\section{CONCLUSÃO}

Em conclusão, o treinamento em ambas as intensidades evitou a deterioração do condicionamento aeróbio imposta pelo avanço da idade, já que os ratos treinados mantiveram constante a intensidade de esforço (velocidade) equivalente à transição metabólica no decorrer do experimento. Por outro lado, os animais mantidos sedentários apresentaram redução da intensidade de esforço equivalente à transição metabólica determinada no final do experimento, se comparada com os achados do teste efetuado pelos animais no início do experimento. Além disso, o treinamento na intensidade superior à MFEL reduziu a produção de lactato pelo músculo esquelético. Isso fica evidente nos resultados de liberação de lactato pelo músculo sóleo isolado, o qual mostrou valores menores pelo grupo TS quando comparado com os grupos TT e Sedentário.

\section{AGRADECIMENTOS}

Os autores agradecem o suporte técnico de Clarice Y. Sibuya, Eduardo Custódio e José Roberto R. da Silva.

Apoio financeiro: Fapesp (proc. 05/58810-1).

\section{REFERÊNCIAS BIBLIOGRÁFICAS}

1. Mcardle WD, Katch FI, Katch VL. Fisiologia do exercício: energia, nutrição e desempenho humano. Editora Guanabara Koogan S.A. Rio de Janeiro. 2008.

2. Wasserman K, Mcilroy MB. Detecting the threshold of anaerobic threshold in cardiac patients during exercise. Pedia Cardio. 1964;20:12-5.

3. Kindermann W, Simon G, Keul J. The significance of the aerobic-anaerobic transition for the determination of work load intensities during endurance training. Eur J Appl Physiol Occup Physiol. 1979;42:25-34.

4. Sjodin B, Jacobs I. Onset of blood lactate accumulation and marathon performance. J Sports Med. 1981;2:23-6.

5. Monod H, Scherer J. The work capacity of a synergic muscular group. Ergonomics. 1985;8:329-38.

6. Mader A, Heck H. A theory of metabolic origin of the anaerobic threshold. Int J Sports Med. 1986;7:45-6.

7. Heck H, Mader A, Hess G, Mucke S, Muller R, Hollmann W. Justification of the 4-mmol/1 lactate threshold. Int J Sports Med. 1985;6:117-30.

8. Gobatto CA, Mello MAR, Sibuya CY, Azevedo JRM, Santos LA, Kokubun E. Maximal lactate steady state in rats submitted to swimming exercise. Comp Biochem Physiol. 2001;130:21-7.

9. Manchado FB, Gobatto CA, Contarteze RVL, Papoti M, Mello MAR. Maximal lactate steady in running rats. JEP Online. 2005;8:4-11.

10. Jacobs I. Blood lactate. Implications for training and sports performance. Sports Med. 1986;3:10-25.

11. Oyono-Enguelle S, Marbach J, Heitz A, Ott C, Gartner M, Pape A, Vollmer JC, Freund H. Lactate removal ability and grated exercise in humans. J Appl Physiol. 1990;68:905-11

12. Gobatto CA. Metabolismo glicídico em músculo sóleo isolado de ratos desnutridos e recuperados. Efeito dos ácidos graxos livres e do treinamento físico. Tese de Doutorado - UNICAMP. Campinas; 1997.

13. Hill AV, Long CNH, Lupton H. Muscular exercise, lactic acid, and the supply and utilization of oxygen: parts IV-VI. Proc Roy Soc B. 1924;97:84-138.
14. Beneke R. Anaerobic threshold individual anaerobic threshold and maximal lactate steady state in running. Med Sci Sports Exerc. 1995;27:863-671.

15. Beneke R. Methodological aspects of maximal lactate steady state-implications for performance testing. Eur J Appl Physiol. 2003;89:95-9.

16. Billat VL, Siverent P, Py G, Korallsztein JP, Mercier J. The concept of maximal lactate steady state: a bridge between biochemistry, physiology and sport science. Sports Med. 2003;33:407-26.

17. Contarteze RVL, Manchado FB, Gobatto CA, Mello MAR. Stress biomarkers in rats submitted to swimming and treadmill running exercises. Comp Biochem Physiol. 2007;A:1-8.

18. Wasserman K. The anaerobic threshold: definition, physiological significance and identification. Adv Cardiol. 1986;35:1-23.

19. Gobatto CA, Kokubun E, Sibuya CY, Mello MAR. Efeitos da desnutrição proteica - calórico e do treinamento físico na produção de ácido lático em ratos machos adultos após testes de cargas progressivas. Resultados preliminares. Ciência e Cultura. 1991;43:725-36.

20. Donovan CM, Brooks GA. Endurance training effects lactate clearance, not lactate production. Am J Physiol. 1983;244:E83-E92.

21. Holloszy JO, Coyle E F. Adaptations of skeletal muscle to endurance exercise and their metabolic consequences. J Appl Physiol: Respiratory, Environmental and Exercise Physiology, Bethesda. 1984;56:831-8.

22. Stanley WC, Gertz EW, Wisneski JA, Neese RA, Brooks GA. Systemic lactate kinectis during exercise graded exercise man. Am J Physiol. 1985;249(6 Pt 1):E595-602

23. Brooks GA. Current concepts in lactate exchange. Med Sci Sports Exerc. 1991;23:895-906.

24. Brooks G A. Intra- and extra-cellular lactate shuttles. Med Sci Sports Exerc. 2000;32:790-9.

25. Jacobs I. Blood lactate. Implications for training and sports performance. Sports Med. 1986;3:10-25.

26. Roth DA. The sarcolemmal lactate transporter: transmembrane determinants or lactate flux. Med Sci Sports Exerc. 1991;23:925-34. 